\title{
FONDI CONSULTED (AND ABBREVIATIONS)
}

All these files are located at the Archivio di Stato in Florence.

Acquisti e Doni

Archivio della Repubblica (Arch. della Rep.)—Signori e Collegi Arti-Arte del Cambio

Auditore delle Riformagioni (Aud. delle Rif.)

Capitani di Parte Guelfa

Carte Alessandre

Carte Strozziane (CS)

Depositeria Generale (DG)

Libri Fabarum

Magistrato Supremo (MS)

Manoscritti (Mano.)

Manoscritti e Codici Litterari (Mano. e Cod. Litt.)—Bardi

Mediceo (Principato) (Med.)

Mercanzia

Miscellanea Medicea

Miscellanea Repubblicana (Misc. Rep.)

Monte di Pietà (MP)

Monte di Pietà del Bigallo

Otto di Guardia e Balìa

Otto di Pratica (Principato)

Pratica Segreta (PS)

Provvisioni (Prov.)

Pupilli

Senato de' Quarantotto

Sindaci (Sin.) 
